\title{
Molecular psychiatry: moving away from one-polymorphism genetic association studies
}

Molecular Psychiatry (2003) 8, 247. doi:10.1038/

sj.mp.4001330

Molecular Psychiatry receives now more than one new manuscript a day. We can only publish a fraction of the articles we receive. We therefore have to be selective in what we choose to publish. Our editorial goal is to be the best source of high-quality translational studies in the field of psychiatry. To achieve that goal, we publish papers in a variety of fields from fundamental molecular biology to imaging, pharmacology, and clinical research. Genetics has been a key area for us. In order to continue to publish some of the most exciting papers in psychiatric genetics, it has become necessary to develop guidelines for the submission of such articles.

Single gene association studies have yielded interesting data, including the findings of apolipoprotein-E gene variations in Alzheimer's disease, and dopamine 4 receptor repeats in attention-deficit hyperactivity disorder. ${ }^{1-3}$ Nevertheless, the field and our methods have greatly improved, so it is really no longer justifiable in contemporary science to use a sample set to examine one single polymorphism, unless there is an extraordinarily compelling reason to do so.

Research groups around the world have in their freezers collections of samples for various psychiatric diagnoses. The cost of genotyping has gone down substantially, and feasibility of testing is not an issue. Psychiatric disorders are all of unknown cause. There is lack of identified and universally agreed pathophysiologic pathways from which a small number of strong candidates can emerge. For this reason, any gene that is expressed in the brain, can, with reasonable explanation, become a candidate for any psychiatric disorder. It is just not credible that having collected well-characterized samples, authors only looked at the single polymorphisms that are reported in many of the papers that we receive. It is far more likely that authors looked at multiple candidates and only report the most significant results, with hypotheses developed a posteriori to justify looking at that specific gene. While we have several one-polymorphism papers in press and in review, with publication scheduled in future issues, we will from now on be extremely rigorous, when we consider that kind of association study.

Most single-polymorphism genetic association studies submitted for publication in Molecular Psychiatry will be rejected without review. The onus is now on the author to provide truly compelling evidence justifying peer-review of such articles. There must also be highly credible justification of the rationale for examining just one SNP in one gene.

On the other hand, we encourage the submission of studies that conclusively examine multiple candidate genes in relevant biological systems. Such studies would examine a variety of polymorphisms and would ideally include haplotypes. It seems that psychiatric disorders may be caused by a combination of the effects of multiple gene alleles whose products interact with the environment to result in psychiatric phenotypes. For that reason, we are also very interested in studies of gene-gene interactions, as well as in studies of gene-environment interactions.

We believe that by using our space to publish more complete studies that examine an entire biological system or that shed new light on the interactions among multiple genes (or between genetic and environmental factors), we will foster progress and stimulate researchers to generate more rigorous data sets that will contribute to advance the field of Molecular Psychiatry.

J Licinio

UCLA Laboratory for Pharmacogenomics E-mail: licinio@ucla.edu

1 Strittmatter WJ et al. Proc Natl Acad Sci USA 1993; 90: 1977-1981. 2 Corder EH et al. Science 1993; 261: 921-923.

3 LaHoste GJ et al. Mol Psychiatry 1996; 1: 121-124. 